\title{
Bile Duct Injuries with Loss of Confluence
}

\author{
Oscar Chapa-Azuela ${ }^{1}$, Alejandro José Rosales ${ }^{1}$, Carmen Roca-Vasquez ${ }^{1}$, \\ Brenda Arcos-Vera ${ }^{2}$, Jorge Alberto Roldan-Garcia ${ }^{1}$, Gustavo Alain Flores-Rangel ${ }^{1, \text { * }}$ \\ ${ }^{1}$ General Hospital of México, Mexico City, México \\ ${ }^{2}$ Issemym Toluca Medical Center, Toluca, Mexico
}

Email address:

gustavoflores_@hotmail.com(G. A. Flores-Rangel)

*Corresponding author

\section{To cite this article:}

Oscar Chapa-Azuela, Alejandro José Rosales, Carmen Roca-Vasquez, Brenda Arcos-Vera, Jorge Alberto Roldan-Garcia, Gustavo Alain Flores-Rangel. Bile Duct Injuries with Loss of Confluence. International Journal of Gastroenterology. Vol. 2, No. 1, 2018 , pp. 7-11. doi: $10.11648 /$ j.ijg.20180201.12

Received: April 12, 2018; Accepted: May 10, 2018; Published: May 30, 2018

\begin{abstract}
Introduction: Iatrogenic bile duct injuries (IBDI) with loss of confluence are understood as those where right and left hepatic ducts lose continuity with the common biliary tree. These represent $4 \%$ of all IBDI and are considered a very demanding surgical challenge. Study design: This is a series of case in a reference center during an eight-year period (2008 2016), where all patients with IBDI and loss of confluence submitted to any bilioenteric derivation procedure were included. Results: From a total of 11 cases, 10 of them (90.1\%) were treated with double bilioenteric derivation and $1(9 \%)$ with a neoconfluence. In $90.9 \%(n=10)$ of the patients a percutaneous catheter of biliary drainage was placed before the surgical procedure. Within a 34.5 months follow-up, the initial approach was successful in $54.5 \%(\mathrm{n}=6)$, meanwhile accumulated achievement was $81.8 \%(n=9)$ considering dilatation and remodeling procedures. From this, $18.2 \%(n=2)$ are still with stenosis of derivation in a dilatation protocol with percutaneous catheter. Conclusions: Double hepatojejunostomy with transanastomotic stents and management of eventual stenosis with percutaneous dilatation as a first therapeutic intention results in a standardized practice that leads to reasonable results compared with other high volume centers.
\end{abstract}

Keywords: Lesion, Bile, Loss, Confluence

\section{Introduction}

Iatrogenic bile duct injuries (IBDI) with loss of confluence are understood as those where right and left hepatic ducts lose continuity with the common biliary tree, correspond to Strasberg E4 injuries and represent a clinical complex scenario due to the intrahepatic ducts route, it's small diameter and high association with vascular affection $(60 \%$ a 78\%)[1-4]. This lesions could happen after an unusual low confluence, secondary to ischemia, or to thermic damage [1].

General incidence of complex biliary lesions secondary to cholecystectomy is between 0.3 to $0.6 \%$ [1], and of these around $4 \%$ will be with loss of confluence. On the other hand, difficulties to repair these lesions lead to a higher proportion of them in reference centers $(9.4 \%$ to $15 \%)$ [5-7].

Due to its low frequency as to the difficulty to develop protocols that contrast possible strategies to repair them, recommendations for its approach come from case series with modest casuistry in general. Maybe the highest experience is the reported by Mercado et al [1] with 53 cases in 18 years, and $88 \%$ cumulative success, recommending in order of preference a derivation by neo-confluence, followed by a double bilioenteric anastomosis and Kasai portoenterostomy; eventually higher hepatic resections should be required or even a liver transplant.

Due to the high technical demand required to repair them, they should be performed in high volume centers with an experienced and multidisciplinary biliary surgical team.

\section{Method}

This study is a series of cases obtained from a prospective database in a HPB surgery specialty center within an 8 year period, from August 2008 to July 2016.

All patients diagnosed with IBDI with loss of confluence determined transoperatively and treated with any bilioenteric derivation were included.

It was considered as initial successful management those 
cases in which the bilioenteric anastomosis remains permeable for a period of time greater than one year without instrumentation or postsurgical manipulation, otherwise, those where anastomosis permeability was achieved after any instrumental or postsurgical biliary manipulation procedure (surgical remodeling or percutaneous catheter) due to a first attempt to repair that development were considered as cumulative success.

Diagnostic approach of suspected IBDI patients consisted of an image study to evaluate collections (abdominal tomography or ultrasound), if there were found, were drained through a percutaneous catheter, In case the above is not adequately achived, laparotomy drainage was performed. Eventual sepsis status was controlled by antibiotic therapy and finally a direct cholangiography was performed using a percutaneous transhepatic biliary catheter towards the bile duct of higher caliber. Occasionally a catheter in both right and left sides was placed.

As a choice procedure, our team preference is double anastomosis with separate sutures, external knot and monofilament suture material of slow absorption caliber 5-0 (PDS) towards a desfunctionalized Roux-en-Y jejunal loop. If the patient's condition was adequate (without sepsis and without intra-abdominal collections), an immediate derivation surgery was made, otherwise, procedure was delayed to a late repair which time depended on each particular case.

Postsurgical care, if the patient's conditions were appropiate, included prophylactic antibiotic therapy with double scheme (metronidazole/ ciprofloxacin) based on local susceptibility, and liquid diet in the first 24 hours. Percutaneous catheter is usually advanced until the anastomosis was traversed and removed on average 3 to 4 weeks after.

Perioperative complications were considered in the first 90 days. Biliary derivation stenosis were suspected in patients with clinically manifested jaundice or with serum elevation of direct bilirubin, then a hepatobiliary ultrasound was performed to confirm intrahepatic biliary dilatation followed by a percutaneous catheter placement $8.5 \mathrm{fr}$ caliber on the biliary segment with the highest caliber, through which a direct cholangiography and cholestasis palliation was achieved, to then move toward the jejunum overpassing the stenosis site; in case of getting it, a trimestral dilatation protocol was initiated with $2 \mathrm{fr}$ per session up to a variable caliber, generally $16 \mathrm{fr}$. On the opposite, whether overpass the stenosis site was not possible or it relapses after a dilatation protocol, patient underwent a surgical remodeling, dismantling the previous anastomosis and building a new one.

\section{Results}

In the period of time considered for the present study, a total of 78 bile duct complex injuries were repaired (Strasberg E:1 - E5), from them, 11 (14.1\%) were injuries with loss of confluence.
Mean age was 34.7-years (17 to 59), with a female prevalence $(\mathrm{n}=8 / 72.7 \%)$. Mean period between index and repair surgeries was 139 days (from 3 to 308) and follow-up was 34.5 months ( 7 to 144 ).

In $90.9 \%(n=10)$ of the patients a percutaneous catheter of biliary derivation was decided to be placed before surgical procedure, 7 of them went towards the right hepatic duct, 1 towards the left, and 2 towards both ducts. In $27.3 \%(n=3)$ of the patients, interval surgeries were performed between index surgery and biliodigestive derivation with hemostasis and surgical drainage of intra-abdominal infected collections, in an average of 2 procedures per patient. Most of the cases were caused by open cholecystectomies $(63.6 \% n=7)$, and less frequently by laparoscopic $(18.2 \% / \mathrm{n}=2)$ or laparoscopic turned into open $(18.2 \% / \mathrm{n}=2)$ approaches.

In 10 cases $(91 \%)$ a double bilioenteric derivation was decided and only in 1 patient a neo-confluence was made $(9 \%)$. Bleeding was $667 \mathrm{ml}$ in average and surgical time was $333 \mathrm{~min}$. Four patients $(36.4 \%)$ required transfusions of red cell concentrates $(2$ patients required 2 concentrates and 2 patients required 1). Hospital stay was in average 10.2 days and complications' incidence was $27.3 \%(n=3)$. Reoperations were not required within the first 90 days and no deaths were observed (table 1).

Table 1. 90-day results.

\begin{tabular}{ll}
\hline Complications & $\mathrm{n}=3(27.3 \%)$ \\
Biliary fistula & $n=1$ \\
Cholangitis & $n=1$ \\
Surgical site abscess & $n=1$ \\
Days of hospital stay & 12.2 días (4 a 32 días) \\
Reoperations & $\mathrm{n}=0$ \\
Mortality & $\mathrm{n}=0$ \\
\hline
\end{tabular}

During long term observation (table 2), 5 patients presented biliary stenosis $(45.4 \%)$, from these, 3 cases were managed with progressive dilatations through a percutaneous catheter; one case was considered successful (9.1\%) with catheter removal whitout clinical or biochemical cholestasis, and 2 cases $(18.2 \%)$ are still in dilatation process. The other 2 patients whit stenosis $(18.2 \%)$ were managed with dismantling and surgical remodeling, because the percutaneous catheter fails to cross the stenosis; in the last review there were no signs of cholestasis.

Table 2. Long-term results (>90 DAYS).

\begin{tabular}{lll}
\hline Result & $\mathbf{n}=$ & $\mathbf{\%}$ \\
\hline Cholangitis * & 1 & $9 \%$ \\
Cholangitis + liver abscess * & 1 & $9 \%$ \\
Stenosis & 5 & $45.4 \%$ \\
Management with dilatation by percutaneous catheter & 3 & $27.3 \%$ \\
Management with remodeling & 2 & $18.2 \%$ \\
\hline
\end{tabular}

* patients who presented cholangitis belong to the same group of patients with stenosis

Within stenosis group, 1 patient presented a cholangitis episode (9\%), and another one presented an episode of cholangitis whit liver abscess (9\%), both cases resolved with antibiotic therapy and replacement of percutaneous 
drainages.

In $81.8 \%$ of the cases $(n=9)$ success was found at a mean follow-up of 34.5 months (table 3), from which $54.5 \%$ were with initial bilioenteric derivation procedure $(n=6), 18.2 \%$ required surgical remodeling $(\mathrm{n}=2)$ and $9.1 \%$ required dilatation procedures $(n=1)$. Meanwhile, $18.2 \%(n=2)$ of the patients are currently with stenosis in dilatation protocol with percutaneous catheter, from these, one patient with 1 catheter (to the right bile duct) and another with 2 catheters (right and left ducts).

\section{Table 3. Actual state}

\begin{tabular}{lll}
\hline Successful management ${ }^{*}$ & 9 & $81.8 \%$ \\
Bilioenteric derivation & 6 & $54.5 \%$ \\
Bilioenteric derivation + surgical remodeling & 2 & $18.2 \%$ \\
Bilioenteric derivation + percutaneous dilatation & 1 & $9.1 \%$ \\
Stenosis & 2 & $18.2 \%$ \\
In dilatation protocol with percutaneous catheter & 2 & $18.2 \%$ \\
\hline
\end{tabular}

\section{Discussion}

Even when advances in interventional radiology have enabled conservative management in some cases of biliary stenosis through percutaneous dilatation procedures (success from $35 \%$ to $85 \%$ )[8], experience reported in this approach about lesions with loss of confluence is limited, moreover it is a procedure just for cases where biliary continuity has not been lost completely. For this reason, the standard approach is through a bilioenteric derivation surgery which could be reached with a Kasai portoenterostomy, a double anastomosis or making a neo-confluence (Figure 1).

Regardless of series of cases with portoenterostomy associated with good results [2], most authors consider this technique the option with the worst long term prognosis, and is reserved as the last election when it is impossible to perform any duct-to-mucosal derivation variant [1], then, neo-confluence and double anastomosis are preferred whenever possible $[1,9]$.

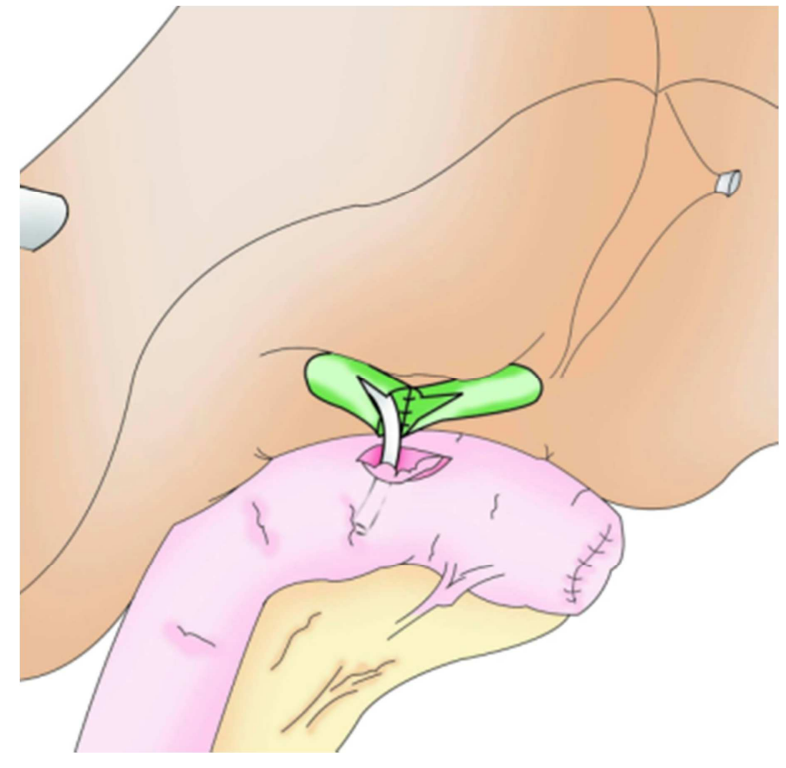

a

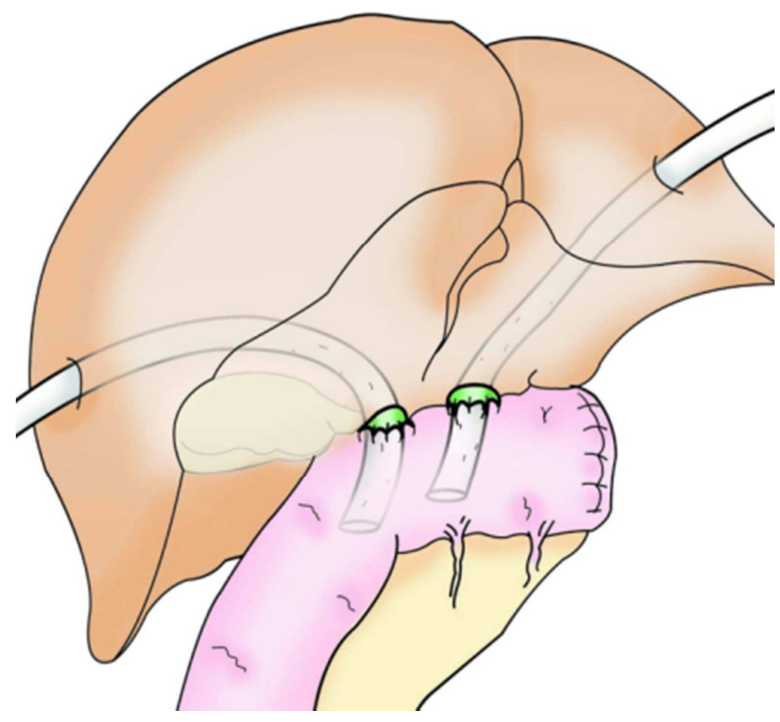

b

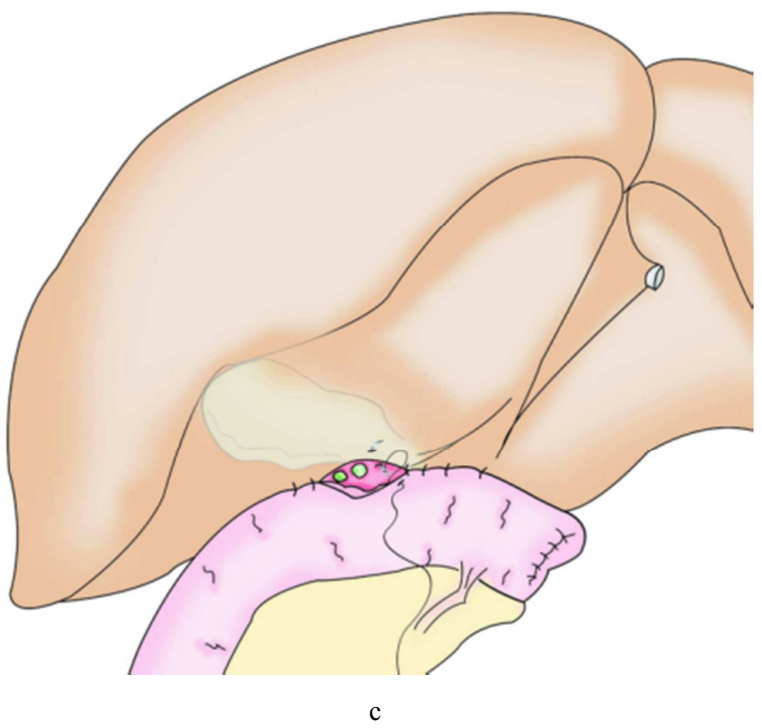

Figure 1. a: Neoconfluence; $b$ : Double derivation; c: Portoenterostomy.

Hepatic resections could eventually be required after several attempts of repair that evolve to stenosis and/or unilateral atrophy, repeated abscess or cholangitis [10]. Moreover, hepatic transplant becomes a better choice when hepatic cirrhosis has developed $[1,8]$.

It should be considered that vascular affection in these kind of lesions lead to a great impact in patient's prognosis in contrast to those with lower biliary lesions, since the general vascular lesions incidence is 30\% [11] (usually of the right hepatic artery), and from these ones just $10 \%$ would be clinically relevant, in cases of compromised confluence the reported incidence is $60-78 \%[2-4]$, representing the main factor associated with failure of a bilioenteric derivation procedure, and with the need of hepatic resections and transplants [11-14]. A possible reason might be because when confluence remains intact in the presence of a right hepatic artery lesion, transverse marginal artery and capillary vessels plexus are able to compensate blood supply from the left hepatic artery to the right bile duct, but when confluence 
is damaged, the transverse marginal artery -which is above the confluence - is at risk of injury, and therefore devascularize right bile duct (figure 2).

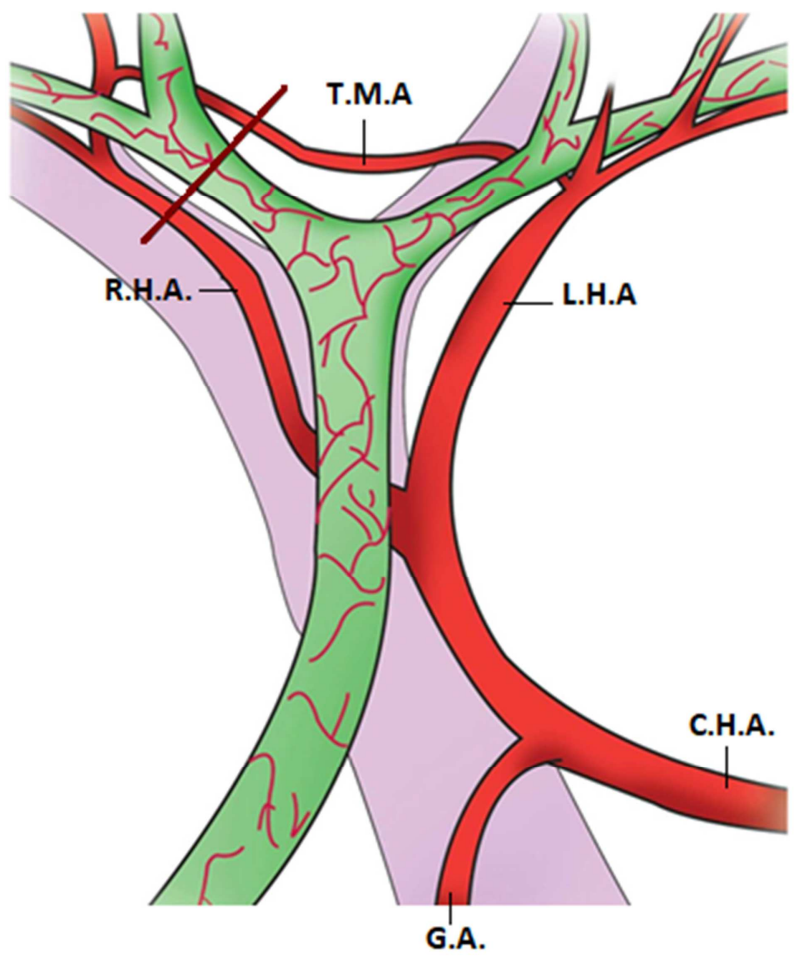

Figura 2. Irrigation of the bile duct / C. H. A: Common Hepatic Artery / G. A: gastroduodenal artery / L. H. A: left hepatic artery / R. H. A: Right Hepatic Artery / T. M. A: Transverse Marginal Artery. In the presence of a lesion of the right hepatic artery, the transverse marginal artery preserves the bile blood flow from the left. If the latter is also injured during intrahepatic dissection of the ducts (red line) ischemia is present.

Different opinions exist related to the best moment to carry on the repair, Strasber [9] has suggested to wait 3 months in lesions with confluence lost meanwhile ischemia is delimit, as lesion is always higher than originally appreciated. In the same way, Santibañez [15] considered that repairing should wait 6 to 8 weeks in order to allow inflammation to be limited; on the contrary, recent reports like the ones from Stilling [16] or Kirk [17] concluded that the repair moment of a bilioenteric derivation by itself, did not modify the long term result. Stewart and Way [18] in a multivariate analysis on 307 patients, revealed that a derivation success depends on complete eradication of the abdominal infection, identification of the lesion level through a cholangiography, adequate surgical technique and an experienced biliary team; when these objectives are achieved, repair could be performed at any time with the expectation of a favorable result without any reason to delay the procedure for an arbitrary time [18].

Once these goals are achieved, patient could be submitted to a repair surgery, where dissection should be so carefully done without compromising vascular supply, but enough to allow debriding fibrosis zones and ensure an anastomosis among healthy tissues $[9,15]$. A transoperative cholangiography could be advisable when considering that the real level of the lesion is not always well correlated with the estimated during initial evaluation. Resecting segments IV and $\mathrm{V}$, coined on the biliary tree is very useful [1, 8] although occasionally it is not required if viable ducts are beyond the liver edge [15].

Identify the main ducts results complicated due to their intrahepatic situation and heavy fibrosis usually presented. The left duct becomes the first objective due to a more horizontal and superficial trajectory below segment 4 . The right duct instead, follows a more vertical trajectory and gets in the liver depth at the base of segment 5, in this case identification becomes easier when considering that both ducts course in the same coronal plane, so that when identifying the left duct trajectory the position of right duct could be inferred. With the left duct identified, dissection continues from left to right with the tip of the clamps in right angle form the same coronal plane of the left duct until finding and dividing the hepatic capsule at cystic plate level, which should be divided to allow opening right pedicle in its sheath, inside which right hepatic duct is found. This is known as Hepp Couinad approach [9, 15].

Once dissected, both ducts are opened on their anterior side at least $1 \mathrm{~cm}$ (if possible) to finally widen the surface of the future anastomosis, ensuring the edge of the incision with a suture point $6-0$. Sometimes, due to the level of the lesion and anatomical variations, this opening on the anterior side is not possible, so, anastomosis is performed using the duct terminal end. Once bile ducts are prepared, a dysfunctional jejunal loop becomes closer in a Y-Roux. Our team preference is a double anastomosis as a first election procedure, because in these cases vascular supply could be severely compromised and an excessive dissection to free the ducts in order to get closer without tension could cause bleeding, whose hemostasis may be a risk of ischemia by injury the vascular flow from left to right.

This behavior that aims to preserve the irrigation, may represent a risk of stenosis due to the reduced caliber of the anastomoses, which is reflected in our high incidence of stenosis, but no case of manifest ischemia.

It is considered that a good quality anastomosis is free of tension, with a wide diameter, with the correct suture material, performed in healthy bile ducts (without fibrosis) and correctly perfused $[19,20$.

Preoperative placement of percutaneous transhepatic catheters are a useful tool to be considered and a standardized practice in our team, advantages include cholangitis management (if so), it allows good quality cholangiographies as many times as needed, enables recognition of bile ducts during dissection and helps in fistula control. Some groups consider its routinely use in IBDI with loss of confluence [9], while others prefer reserve them just for cholangitis cases or inability to establish the level of the lesion [1].

Once bilioenteric derivation is done, this catheter could advance to the gut crossing the anastomosis, be placed just on biliary segment or be removed. Our team prefers to advance it and cross the anastomosis, althoug now it is well known that transanastomotic stents are not the best choice for 
bilioenteric derivations in general, in the specific case of lesions with loss of confluence where duct caliber is limited, its use is frequent [8], because it reduces the incidence of cholangitis and improve patient's evolution [1]. Proper time to remove them has not been defined, reported vary from 3 weeks [9] to 84 months [1].

\section{Conclusions}

Based on the present series of cases, it can be concluded that double hepatojejunostomy as a bilioenteric derivation procedure with transanastomotic stents, and management of eventual stenosis with percutaneous dilatation as a first therapeutic intention and surgical remodeling in case of failure of the latter, results in a standardized practice translated to acceptable results, in agreement to the reported by other high volume centers [1].

\section{References}

[1] Mercado M, Vilatoba M, Contreras A, Leal P, Cervantes E, Arriola $\mathrm{J}$, et al. Iatrogenic bile duct injury with loss of confluence. World J Gastrointest Surg 2015; 7 (10):254-260.

[2] Pickleman J, Marsan R, Borge M. Portoenterostomy. An Old Treatment for a New Disease. Arch Surg. 2000; 135:811-817.

[3] Stewart L, Robinson TN, Lee CM, Liu K, Whang K, Way LW. Right hepatic artery injury associated with laparoscopic bile duct injury: incidence, mechanism, and consequences. J Gastrointest Surg. 2004; 8:523-530.

[4] Laurent A, Sauvanet A, Farges O, Watrin T, Rivkine E, Belghiti J. Major hepatectomy for the treatment of complex bile duct injury. Ann Surg. 2008; 248:77-83.

[5] Gao Z, Li P, Chen F, Tan D. The Clinical Analysis of Bile Duct Injury during Laparoscopic Cholecystectomy Int Jour of Clin Med. 2015; 6:825-830.

[6] Cui Y, Zhang H, Cui N, Li Z. Surgical treatment for benign biliary strictures: single-center experience on 64 cases. EXCLI Jour. 2012; 11:390-398.

[7] B. Melton, MD, Keith D. Lillemoe, MD, John L. Cameron, MD, Patricia A. Sauter, CRNP, JoAnn Coleman, CRNP, and Charles J. Yeo, MD. Major Bile Duct Injuries Associated With Laparoscopic Cholecystectomy. Anns Surg. 2002; 235 (6):888-895.

[8] Blumgart's. Surgery of the Liver, Biliary Tract and Pancreas. 5ta ed, Vol 1, ed elsevier. 2012.
[9] Strasberg SM, Picus DD, Drebin JA. Results of a new strategy for reconstruction of biliary injuries having an isolated rightsided component. J Gastrointest Surg. 2001; 5:266-274.

[10] Perini M, Herman P, Montagnini A, Jukemura J, Coelho F, Kruger J, Bacchella T, Cecconello I. Liver resection fo the treatment of post. cholecystectomy biliary stricture with vascular injury. Wolrd Journal og Gastroenterology. 2015 21; 21 (7):2102-2107.

[11] Strasberg S, Scott W, An analytical review of vasculobiliary injury in laparoscopic and open cholecystectomy. HPB. 2011; 13:1-14.

[12] Perini M, Herman P, Montagnini A, Jukemura J, Coelho F, Kruger J, et al. Liver resection for the treatment of postcholecystectomy biliary stricture with vascular injury. World J Gastroenterol 2015; 21 (7):2102-2107.

[13] Li J, Frilling A, Nadalin S, Broelsch C, Malago M. Timing and Risk Factors of Hepatectomy in the Management of Complications Following Laparoscopic Cholecystectomy. J Gastrointest Surg. 2012; 16:815-820.

[14] Laurent A, Sauvanet A, Farges O, Watrin T, Rivkine E, Belghiti J. Major Hepatectomy for the Treatment of Complex Bile Duct Injury. Annal of Surg. 2008; 248 (1):77-83.

[15] Santibañee E, Ardiles V, Pekolj J. Complex bile duct injuries: management. HPB 2008. 10; 4-12.

[16] Nicolaj M. Stilling1, Claus Fristrup1, André Wettergren2, Arnas Ugianskis3, Jacob Nygaard4, Kathrine Holte2, Linda Bardram2, Mogens Sall3 \& Michael B. Mortensen1. Longterm outcome after early repair of iatrogenic bile duct injury. A national Danish multicentre study. HPB 2015, 17, 394-400.

[17] Kirks R, Barnes T, Lorimer P, Cochran A, Siddiqui I, Martinie J, Baker E, Iannitti D, Vrochides D. Comparing early and delayed repair of common bile duct injury to identify clinical drivers of outcome and morbidity. HPB 2016; 18:718-725.

[18] Stewart L, Way L. Laparoscopic bile duct injuries: timing of surgical repair does not influence success rate. A multivariate analysis of factors influencing surgical outcomes. HPB 2009:11; 516-522.

[19] Mercado M, Arriola J, Dominguez I, Elnecavé A, Urencio M, Ramirez F, et al. Lesión iatrogénica de vía biliar con pérdida de confluencia: Opciones quirúrgicas. Cir Gen. 2010; 32 (3):160-166.

[20] Jabłońska B, Lampe P. Iatrogenic bile duct injuries: Etiology, diagnosis and management. World J Gastroenterol. 2009; 15 (33):4097-4104. 